\title{
Disordered periodic systems at the upper critical dimension
}

\author{
R. Chitra \\ Physics Department, Rutgers University, Piscataway, NJ-08854, USA \\ T. Giamarchi \\ Laboratoire de Physique des Solides, CNRS-URA 02, UPS Bat. 510, 91405 Orsay France \\ P. Le Doussal \\ CNRS-Laboratoire de Physique Theorique de l'Ecole Normale Superieure, 24 rue Lhomond,75231 Cedex 05, Paris, France.
}

\begin{abstract}
The effects of weak point-like disorder on periodic systems at their upper critical dimension $D_{c}$ for disorder are studied. The systems studied range from simple elastic systems with $D_{c}=4$ to systems with long range interactions with $D_{c}=2$ and systems with $D_{c}=3$ such as the vortex lattice with dispersive elastic constants. These problems are studied using the Gaussian Variational method and the Functional Renormalisation Group. In all the cases studied we find a typical ultra-slow $\log \log (x)$ growth of the asymptotic displacement correlation function, resulting in nearly perfect translational order. Consequences for the Bragg glass phase of vortex lattices are discussed.
\end{abstract}

\section{INTRODUCTION}

The study of the physics of elastic systems in the presence of disorder has long been a subject of great the retical and experimental interest, with applications to systems as diverse as charge density waves, Wigner crystals, magnetic bubbles $\mathbf{B}_{\text {, }}$ etc. . These studies were given a further impetus with the discovery of high-Tc superconductors which were found to be of the Type II kind. The resolution of the various theoretical challenges posed by these disprdered vortex lattices 0 is crucial for the technological applications of high- $T_{c}$ materials. Early studies by Larkin 6 of the elastic system with point-like disorder indicated that disorder is relevant and that perfect long range translational order is destroyed below four dimensions. Since for a periodic system thermal effects dominate over that of disorder in dimensions $d<2, d=4$ and $d=2$ are the upper and lower critical dimension for the relevance of disorder. However, the approximate model of independent random forces acting on each vortex line used in Ref. 6.7 was insufficient to obtain the asymptotic properties of the disorder dominated phase. Scaling arguments 8 suggested, within a purely elastic description, a slower, logarithmic growth of displacements. However, general arguments also tended to prove that disorder always favors the presence of dislocations 10 which thus invalidates the elastic approximation and leads to a destruction of the translational order. Although the precise nature of the disordered phase was a subject of much debate there was a thus a consensils that translational order was absent and that disorder leads to a glassy state with diverging barriers and pinning10.11. Various experimental features such as the existence of a first order transitign 12 . 23 , presence of large dislocation free regions in decoration experiments 14 , and termination of the melting line 33.5 could not be explained by these theories.

A quantitative theory of the elastic vortex lattice with point disorder was recently developed 16,17 using both Functional Renormalization Group (FRG) and Gaussian Variational methods (GVM). These methods were originally introduced to study random manifold systems 182 . In the case of periodic systems both methods were found to be in qualitative and quantitative agreement, the FRG heing precise in $d=4-\epsilon$ dimensions and the variational method being applicable for $2 \leq d<4$. These studies 16 , 17 yielded a description of the vortex lattice at all scales and demonstrated that while disorder produces alsepricigrowth of displacements at short length scales, periodicity changes the growth to logarithmic at large scales order. However, due to the abovementioned energy arguments indicating 9.10 that disorder always generate dislocations, it was important to determine whether these results, derived in the elastic approximation would apply to realistic systems. These energy arguments, reexamined in Ref. 17 turned out to be incorrect and instead it was found that dislocations are unfavourable for weak disorder in $d=3$. This led to the prediction of a distinct thermodynamically stable dislocation free glass phase called the Brago o lass because of its nearly perfect translational order and perfect topological order. Furthermore it was proposed12722 that the phase seen experimentally at low fields was the Bragg glass, which accounted paturally for the first-order transition and the decoration experiments. These predictions were confirmed by numerical23 25 and analytical26 33 studies.

These early studies were made using dispersionless elastic constants, an approximation indeed valid to study the large scale properties of the system. However, in systems such as high $T_{c}$ superconductors the penetration depths can be large compared to the lattice constant, resulting in the possibility of non-local elastic constants at intermediate 
length scales. The effect of such non local elasticity was qualitatively studied in Ref. 5,17 and in more detail recently in Ref. 34,35 where the Larkin length and the pinning force were computed, using a renormalization group procedure.

In view of the physical importance and the remarkable properties of the Bragg glass phase, it is interesting to compute other physical properties, such as the positional order for intermediate length scales where the dispersion of the elastic constants is important. Interestingly, this dispersivity of the elastic constants pushes the $d=3$ Abrikosov lattice to its upper critical dimension 3435 . We thus address in this paper the more general question of the effects of disorder right at the upper critical dimension $D_{c}$ (for a discussion on what happens at the lower critical dimension see Ref. 17,36 39). Other examples of such systems which mimic the behaviour at $d=4$ are single component elastic systems in $d=2$ such as charge density waves where long range $1 / r$ Coulomb interactions increase the effective dimension of the system to $d=4$ i.e., $D_{c}=2$. Other motivations for this problem are theoretical. First, the logarithmic growth of displacements found for $d<4$ has a prefactor proportional to $\epsilon=4-d$. It is thus interesting to study how the displacements grow at the upper critical dimension. Second, it also allows us to check and compare the results of the GVM and the FRG when the disorder is marginal.

The plan of the paper is as follows. Section $\mathbb{1}$ is devoted to the study of simple isotropic elastic systems at the upper critical dimension $d=4$ using both the GVM and the FRG. We compute the correlation functions and the characteristic length of the system, and compare the two methods. In Section III] we study physical systems with dispersive elastic constants and anisotropy, in particular the $d=3$ vortex lattice in the non local elastic regime. The conclusions are presented in Section IV.

\section{ELASTIC SYSTEMS AT THE UPPER CRITICAL DIMENSION $D_{C}=4$}

\section{A. The model}

We consider the simplest model of an isotropic elastic system where deviations from the equilibrium positions, characterised by the variables $u_{\alpha}$, are described in a coarse-grained manner by the following continuum hamiltonian

$$
H=\int d^{d} x\left[\frac{c}{2}\left(\partial u_{\alpha}\right)^{2}+V(x) \rho(x)\right]
$$

Here $d$ is the spatial dimension and the index $\alpha$ runs over the number of components of the variable $u$. Depending on the physical origin of $u$, the above hamiltonian can be used to describe various disordered elastic systems. For example, the field $\mathbf{u}$ could denote the displacements from the equilibrium position for vortex lattices or could be a phase variable in the case of charge density waves. $c$ is the non-dispersive elastic modulus and $V(x)$ is the random potential describing the point-like impurities which couples to the density $\rho$. The random potential is gaussian gorrelated $\left\langle V(x) V\left(x^{\prime}\right)\right\rangle=W \delta\left(x-x^{\prime}\right)$. We use the decomposition of the density in terms of harmonics of the lattice 6.17

$$
\rho(x) \simeq \rho_{0}\left(1-\partial_{\alpha} u_{\alpha}+\sum_{K \neq 0} e^{i K(x-u)}\right)
$$

where $K$ are the vectors of the reciprocal lattice and $\rho_{0}$ is the average density. Using (2) and the replica trick to average over disorder we obtain the following replicated hamiltonian

$$
H_{\mathrm{eff}}=\int d^{d} x \sum_{a} \frac{c}{2}\left(\partial u_{\alpha}^{a}\right)^{2}-\sum_{a, b, K \neq 0} \frac{\rho_{0}^{2} W}{2 T} \cos \left(K \cdot\left(u^{a}(x)-u^{b}(x)\right)\right)
$$

Here $T$ is the temperature, and irrelevant terms above the lower critical dimension have been discarded. To obtain the effects of disorder on all length scales one needs to study the theory retaining all the cosine terms in the replicated Hamiltonian (3). However, since we are mainly interested in the asymptotic physics which is governed by the fundamental $K$ vector $K_{0}$, we retain only the lowest harmonic $K=K_{0}$ in (3). We now study the hamiltonian (3) using both the GVM and the FRG.

\section{B. Gaussian Variational Method}

To solve (3), we follow the lines of Ref. 16,17, and introduce the variational Hamiltonian 


$$
H_{0}=\frac{1}{2} \int \frac{d^{d} q}{(2 \pi)^{d}} \sum_{a b} u_{\alpha}^{a} G_{\alpha \beta}^{-1 a b} u_{\beta}^{b}
$$

The indices $a, b$ denote the replicas and $\alpha, \beta$ denote the components of the field. Here $G$ is the variational Green's function matrix which is parametrised as

$$
G_{a b, \alpha \beta}^{-1}=f_{\alpha \beta}(q) \delta^{a b}-\sigma_{\alpha \beta}^{a b}
$$

$f_{\alpha \beta}(q)=c q^{2} \delta_{\alpha \beta}$ and the $\sigma$ are called the self-energy parameters. Since the disorder induced interaction in $H_{\mathrm{eff}}$ is essentially local, the $\sigma$ 's are all taken to be constants. These parameters are determined by saddle point equations which are obtained by minimising the variational free energy $F_{\text {var }}=F_{0}+\left\langle H_{\text {eff }}-H_{0}\right\rangle_{H_{0}}$ with respect to $\sigma$. The variational equations are (see Ref. 17 for technical details)

$$
\begin{aligned}
G_{c \alpha \beta}^{-1} & =f_{\alpha \beta} \\
\sigma_{\alpha \beta}^{a \neq b}(v) & =\frac{W}{2 T} K_{0 \alpha} K_{0 \beta} e^{\left[-\frac{1}{2} K_{0 \alpha} K_{0 \beta} B_{\alpha \beta}^{a \neq b}(0, v)\right]}
\end{aligned}
$$

where $G_{c \alpha \beta}^{-1}(q)=\sum_{b} G_{\alpha \beta}^{-1 a b}(q)$ and $B$ is defined as

$$
B_{\alpha \beta}^{a b}=T \int \frac{d^{d} q}{(2 \pi)^{d}}\left[G_{\alpha \beta}^{a a}+G_{\alpha \beta}^{b b}-2 \cos (q x) G_{\alpha \beta}^{a b}\right]
$$

There are two kinds of solytions to these equations. One is the replica symmetric and the other is the replica symmetry broken one. Earlier work 16 .17 has shown that the appropriate solution has a replica symmetry broken structure (RSB). We thus parametrize all quantities off-diagonal in the replica indices by hierarchical matrices 19

$$
\begin{aligned}
G^{a a}(q) & =\tilde{G}(q) \\
B^{a a}(x) & =\tilde{B}(x) \\
G^{a b}(q) & =G(q, v)
\end{aligned}
$$

where $0<v<1$ and $B^{a b}(x)=B(x, v)$. The indices $\alpha, \beta$ have been suppressed in the parametrisations presented above.

The saddle point equations (6) now take the form

$$
\sigma_{\alpha \beta}(v)=\frac{W}{2 T} K_{0 \alpha} K_{0 \beta} e^{\left[-\frac{1}{2} K_{0 \alpha} K_{0 \beta} B_{\alpha \beta}(0, v)\right]}
$$

where

$$
B_{\alpha \beta}(0, v)=2 T \int \frac{d^{d} q}{(2 \pi)^{d}}\left[\tilde{G}_{\alpha \beta}(q)-G_{\alpha \beta}(q, v)\right]
$$

Since $B(0, v)$ in (9) is essentially local, it follows that it is isotropic which results in the following simplification i.e., $B_{\alpha \beta}(x=0)=\delta_{\alpha \beta} B(v)$ with $B(v)$ defined below. Using this in (9) we find that $\sigma_{\alpha \beta}(v)=\delta_{\alpha \beta} \sigma(v)$. We now search for a solution for $\sigma$ which has full replica symmetry breaking imbedded in it. This can be done in an easier manner by recasting the equations in terms of a new variable $[\sigma]=v \sigma(v)-\int d u \sigma(u)$. Plugging in the ansatz of RSB, it can be shown that the correct solution for $[\sigma]$ has the following form for $2<d<4[16.17$.

$$
\begin{aligned}
& {[\sigma](v) \propto v^{\frac{2}{\theta}}, \quad v \leq v_{c}} \\
& {[\sigma](v)=\Sigma, \quad v \geq v_{c}}
\end{aligned}
$$

At the upper critical dimension there are corrections to the simple power law. Note that a knowledge of the exponent $\theta$, the break-point $v_{c}$ and $\Sigma$ is sufficient to fix the full functional form of the original $\sigma(v)$. As a first step in evaluating these quantities one uses the the inversion rules for hierarchical matrices 19 to obtain

$$
\begin{aligned}
B(0, v)= & B\left(0, v_{c}\right) \\
& +\int_{v}^{v_{c}} d u \int \frac{d^{d} q}{(2 \pi)^{d}} \frac{2 T \sigma^{\prime}(u)}{\left[G_{c}^{-1}(q)+[\sigma](u)\right]^{2}}
\end{aligned}
$$


where

$$
B\left(0, v_{c}\right)=\int \frac{d^{d} q}{(2 \pi)^{d}} \frac{2 T}{G_{c}^{-1}(q)+\Sigma}
$$

Therefore, using Eqs.(11, 12, 13) in (6) one can obtain a self-consistent solution for $\sigma$. Adapting the general framework presented above to the case of a single component model where $G_{c}^{-1}=c q^{2}$ and using (12) we find that the equation determining $[\sigma]$ is

$$
\sigma(v) \int \frac{d^{d} q}{(2 \pi)^{d}} \frac{K_{0}^{2} T}{\left[G_{c}(q)^{-1}+[\sigma](v)\right]^{2}}=1
$$

Solving for $[\sigma]$, we find

$$
[\sigma](v)=\frac{A v}{\log ^{2} \frac{A v}{c \Lambda^{2}}}
$$

where $\Lambda$ is the ultra-violet momentum cut-off and $4=K_{0}^{2} T S_{d} / 2 c^{\frac{d}{2}}$ where $S_{d}$ is the value of the angular integration in $d$ dimensions. For $d<4,[\sigma]$ is a pure power law 16$][-17$ with the exponent $\theta=d-2$ as shown in (11). The logarithmic corrections are specific to $d=4$ (or rather $d=D_{c}$ as will be seen later) and have important consequences for the physical properties such as the correlation functions. The solution (15) for $[\sigma]$ is valid upto some $v=v_{c}$ above which $[\sigma]$ is a constant. This constant value is again fixed by (9).

Using (7) and (9) we can now calculate the correlation functions. The displacement correlation function is given by

$$
\begin{aligned}
\tilde{B}(x) & =\overline{\left\langle(u(x)-u(0))^{2}\right\rangle} \\
& =2 T \int \frac{d^{d} q}{(2 \pi)^{d}}(1-\cos (q x)) \tilde{G}
\end{aligned}
$$

with

$$
\tilde{G}(q)=G_{c}(q)\left[1+\int_{0}^{1} \frac{d v}{v^{2}} \frac{[\sigma](v)}{\left[G_{c}^{-1}+[\sigma](v)\right]}\right]
$$

Since the long-wavelength behaviour of $\tilde{B}$ is governed by the small $v$ behaviour of $[\sigma]$, the asymptotic displacement correlation function is

$$
\tilde{B}(x)=\frac{2}{K_{0}^{2}} \log \log (\Lambda x)
$$

This growth of the displacements is indeed as expected slower than the logarithmic growth seen below four dimensions

$$
\tilde{B}(x)=\frac{2 m}{K_{0}^{2}} A_{d} \log |x|
$$

with $A_{d}=4-d$ and $m$ is the number of components of $u$.

One can also extract 17 the Larkin length $R_{c}$ given by $R_{c}=\sqrt{\frac{c}{\Sigma}}$ from the variational solution presented above. At zero temperature we obtain

$$
R_{c}=\frac{a}{2 \pi} \exp \frac{2 c^{2} \pi^{2}}{K_{0}^{4} W}
$$

where $a$ is the lattice spacing. The exponential growth, is characteristic of the upper critical dimension and is an extrapolation of the power law form

$$
R_{c}=\left(c^{2} / W K_{0}^{4} c_{d}\right)^{1 /(4-d)}
$$

occurring for $d<4$. The result (21) given by the variational methad_oincides with the naive dimensignal result or the more accurate result for $R_{c}$ obtained using the FRG procedure 34, 35. Thus, as was already the case 16.17 for $d<4$, the variational method gives the correct disorder dependence of the Larkin length. 


\section{Functional Renormalisation Group}

We now compute the correlation functions ysing a functional renormalization group procedure. The results of the GVM and the FRG agree very well for $d<416.17$. As is usual with renormalization procedures, we can expect the FRG to work extremely well at the upper critical dimension. This provides another test to verify whether the equivalence between the FRG and GVM results which exists in $d<4$ persists in $d=4$. For simplicity, here we restrict to a single component model.

We denote by $\Delta\left(u^{a}(x)-u^{b}(x)\right)$ the nonlinear term, proportional to the disorder strength in the hamiltonian (3). For clarity we set $K_{0}=2 \pi$. The function $\Delta(z)$ is initially $\sim \cos (2 \pi z)$ and the idea is to obtain flow equations for this function whose fixed point solutions will allow one obtain the correlation functions. In dimensions $d>4$, the gaussian fixed point $\Delta(z)=0$ is perturbatively stable, and thus the cosine potential $\Delta$ can be expanded around $z=0$. However, for $d \leq 4$, we need to retain all the terms in the expansion because they are all marginal. Hence, to study the flow of all these terms one uses the FRG as opposed to the usual RG. To obtain the flow equations of $\Delta$ and $T$, we use the rescaling $x \rightarrow e^{l} x$ and $u \rightarrow e^{\zeta l} u$. Sipce the potential $\Delta$ is periodic in its argument, $u$ cannot be rescaled, implying $\zeta=0$. Using a momentum-shell $\mathrm{RG}^{\mathrm{LB}}$, we find that the system is described by a zero temperature fixed point. The corresponding flow equations for $\Delta$ and $T$ for $d \leq 4$ is

$$
\begin{aligned}
\frac{d T}{d l} & =(2-d) T \\
\frac{d \Delta}{d l} & =\epsilon \Delta+\frac{1}{2}\left(\Delta^{\prime \prime}\right)^{2}-\Delta^{\prime \prime} \Delta^{\prime \prime}(0)
\end{aligned}
$$

where $\epsilon=4-d$ and corrections to these equations are of higher order in $\epsilon$. A factor $1 / S_{d}=2^{d-1} \pi^{d / 2} \Gamma[d / 2]$ has been absorbed in $\Delta$. From the flow equation for $T$ we see that temperature is an irrelevant variable for all $d>2$. The periodic fixed point solution to this highly non-linear differential equation for $\Delta$, for finite $\epsilon$ is 16 . 17

$$
\Delta^{*}=\frac{\epsilon}{72}\left(\frac{1}{36}-z^{2}(1-z)^{2}\right)
$$

for $z$ in the primary interval $[0,1]$ and is extendable to other values of $z$ by periodicity. The solution in (24) is non-analytic at $z=0$ and this non-analyticity is generic to disordered fixed points of the kind studied here.

As anticipated, (24) shows that at the upper critical dimension the fixed point is $\Delta(z)=0$. Note that the RG equation in this case $(d=4)$ gives the exact large scale behaviour while in $d=3$ one must rely on the $O(\epsilon)$ expansion. To find the scale dependence of the function $\Delta_{l}(z)$ in $d=4$, we use the rescaling

$$
\Delta_{l}^{*}(z)=\frac{y}{l}
$$

Substituting this in (23), the corresponding fixed point equation satisfied by $y$ is

$$
0=y+\left(\frac{1}{2} y^{\prime \prime}\right)^{2}-y^{\prime \prime} y^{\prime \prime}(0)
$$

Since this equation has a structure identical to (23) with $\epsilon=1$ we can use the same solution to obtain

$$
y=\frac{1}{72}\left(\frac{1}{36}-z^{2}(1-z)^{2}\right)
$$

in the interval $[0,1]$. Therefore, (27) together with the rescaling (25) for $\Delta^{*}$ specifies the fixed point solution in $d=4$. This solution also exhibits the afore-mentioned non-analytic behaviour at the origin. Another interesting feature of the solution to (23) in $d=4$ is that the explicit $l$ dependence of the solution shows us how the fixed point solution is approached as one scales the system. Using the above results, we can now calculate the displacement correlation function $B(q)=\left\langle u^{a}(q) u^{a}(-q)\right\rangle$ which satisfies the following flow equation

$$
B(q)=e^{4 l} B\left(q e^{l}, T, \Delta_{l}\right)=\left(\frac{1}{q a}\right)^{4} B\left(a^{-1}, T=0, \Delta^{*}\right)
$$

In (28), we have used $e^{l}=(q a)^{-1}$ where $a$ is the lattice spacing which provides the ultra-violet cut-off. For long wavelength correlations, one can expand (28) perturbatively in $\Delta^{*}$ to finally obtain

$$
B(q)=\left(\frac{1}{q}\right)^{4}\left(-\Delta^{* \prime \prime}(0)\right)
$$


Using (27) and restoring the factor $S_{4}=8 \pi^{2}$ this leads to

$$
B(q)=\frac{8 \pi^{2}}{36} \frac{1}{q^{4} \log (a q)}
$$

The fourier transform of (30) results in the following real space displacement correlation function

$$
\tilde{B}(x)=\overline{\left\langle(u(x)-u(0))^{2}\right\rangle}=\frac{8 \pi^{2}}{36 K_{0}^{2}} \log \log \Lambda x
$$

where we have restored $K_{0}$. We find that both the GVM and FRG methods (19,31) yield the same form for

$$
\tilde{B}(x) \simeq \frac{b}{K_{0}^{2}} \log \log [\Lambda x]
$$

The prefactor $b$ which also determines the exponent in the translation correlation function is $b_{v a r}=2$ and $b_{f r g}=2.19$. This discrepancy in the value of $b$ is the same as the one that exists at dimensions $d<4$. There such a difference was attributed to the fact that the variational method underestimates the effect of fluctuations and one expects the the same argument to hold in $d=4$ as well.

\section{PHYSICAL SYSTEM WITH NON-LOCAL ELASTIC CONSTANTS}

\section{A. Single Component Systems}

Let us now turn to a realistic system with long range interactions. The simplest system is a CDW system with long range Coulomb interactions. In this case the field $u$ is a simple scalar field describing the phase. Due to the long range nature of the Coulomb interaction the elastic propagator becomes 10

$$
G_{c}^{-1}(q)=c q^{2}+d q
$$

instead of the simple $q^{2}$ propagator corresponding to local elasticity. By writing $d q=\alpha(q) q^{2}$ with $\alpha=\frac{d}{q}$, we can view the Coulomb contribution as being equivalent to a non-local elastic modulus. When $d$ is large this shifts the "physical" upper critical dimension from $D_{c}=4$ to $D_{c}=2$. The results of the previous section are thus directly applicable. We find that $[\sigma]$ has the same form as that given by (15) with $A=\frac{2 \pi d^{2}}{K_{0}^{2} T}$. Rather surprisingly, the asymptotic displacement correlation function is found to grow in precisely the same manner as the correlations in $d=4$

$$
\tilde{B}(x)=\frac{2}{K_{0}^{2}} \log \log (\Lambda x)
$$

The FRG can be used in the present case with the replacements $\epsilon=2-d$ and $S_{4} \rightarrow S_{2}$ in (30). This results in the same correlation as in (34) with the prefactor 2.19 instead of 2. Note however, that in systems such as Wigner crystals where transverse displacements exist, these transverse displacements are insensitive to the long range part of the Coulomb interaction and the upper critical dimension remains $D_{c}=444$, leading to a different result for the correlations in $d=2$.

\section{B. Vortex Lattice}

We focus in this section on another physical realization of elastic systems at the upper critical dimension, namely, the vortex lattice in $d=3$. The physical properties of such a system with non-dispersive elastic constants are now well known (see e.g. Ref. 37 and references therein) and lead to the phase diagram of Figure 1. 


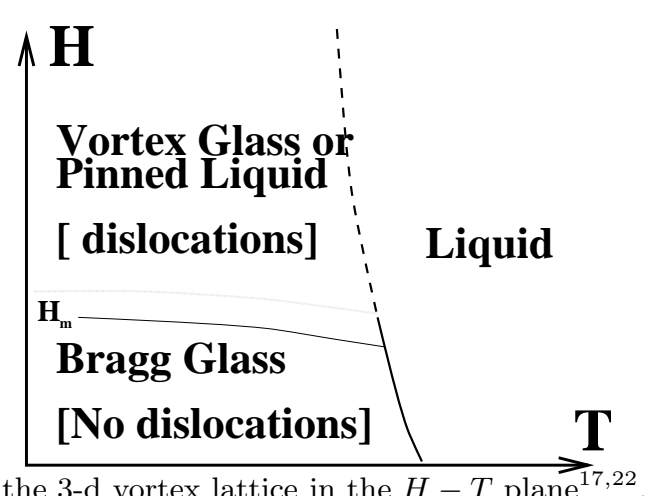

FIG. 1. Schematic phase diagram of the 3-d vortex lattice in the $H-T$ planet, 22 The stability region of the Bragg glass phase in the magnetic field $H$, temperature $T$ plane is shown for moderate fields (the very low field region is not shown). The thick line is expected to be first order, whereas the dotted line should be either second order or a crossover. Upon increasing disorder the field induced melting occurs for lower fields as indicated by the thin solid line.

The Bragg glass occurs for weak disorder where collective pinning works. The system is dislocation free and displacements are logarithmic. At high fields the disorder is stronger and dislocations are present. We study here the effect of non-local elastic constants in the Bragg glass phase. For a realistic form of the dispersion of these moduli i.e., momentum dependence, the system could effectively behave like a $d=4$ system at intermediate lengthscales. This scenario is realised in high- $T_{c}$ vortex lattices.

\section{Model}

The prototype elastic hamiltonian for $d=3$ vortex lattices formed in type II superconductors with point-like disorder is

$$
\begin{aligned}
H= & \frac{1}{2} \int \frac{d^{2} q_{\perp} d q_{z}}{(2 \pi)^{3}}\left[\left(c_{11}-c_{66}\right)\left(q_{\alpha} u_{\alpha}\right)^{2}+c_{66}\left(q_{\alpha} u_{\beta}\right)^{2}\right. \\
& \left.+c_{44}\left(q_{z} u_{\alpha}\right)^{2}\right]+\int d^{2} r d z V(r, z) \rho(r, z)
\end{aligned}
$$

Here $\mathbf{u}$ are the displacement vectors and $\alpha, \beta$ represent the $x, y$ components. In (36), $c_{11}, c_{66}$ and $c_{44}$ are the compression, shear and tilt moduli respectively. These elastic constants are in general momentum dependent and typically depend on the detailed properties of the superconductors. They have been evaluated within a Ginzburg Landau theory and are given by the following expressions

$$
\begin{aligned}
& c_{66}=\frac{\Phi_{0} B}{(8 \pi \lambda)^{2}} \\
& c_{44}=\frac{B^{2}}{4 \pi} \frac{1}{1+\lambda^{2} / \epsilon^{2} q_{\perp}^{2}+\lambda^{2} q_{z}^{2}}+c_{44}^{s} \\
& c_{11}=\frac{B^{2}}{4 \pi} \frac{1+\lambda^{2} / \epsilon^{2} q^{2}}{\left(1+\lambda^{2} q^{2}\right)\left(1+\lambda^{2} / \epsilon^{2} q_{\perp}^{2}+\lambda^{2} q_{z}^{2}\right)}
\end{aligned}
$$

Here $\Phi_{0}$ is the flux quantum, $B$ is the magnetic field along the c axis, $\epsilon=\sqrt{M_{\perp} / M_{z}} \ll 1$ is the anisotropy parameter, $\lambda$ is the London penetration depth in the $a b$ plane and $q_{\perp}$ is the in-plane momentum. $c_{44}^{s}$ is the single vortex contribution which is negligible for $q_{\perp}<1 / a$. Note that though the shear modulus is always non-dispersive, $c_{11}$ and $c_{44}$ are both dispersive, in other words, non-local. The origingf this-dispersion is known to lie in the long ranged

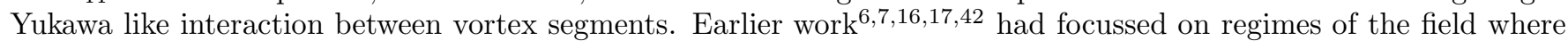
the dispersion in these constants was negligible and one recovered a completely local hamiltonian. A logarithmic growth of the asymptotic transverse and longitudinal displacements was found in this case. This growth was also found to be isotropic despite the obvious anisotropy in the elastic hamiltonian. Apart from this logarithmic regime, a random manifold regime with a power law growth of displacements for intermediate length scales where all the cosine terms were relevant was also found. However, in addition to these regimes, there exists another regime where the dispersive nature of the elastic constants discussed above becomes crucial. This is the region where $c_{11}$ and $c_{66}$ are 
both non-dispersive with $c_{11} \gg c_{66}$ and $c_{44}$ is dispersive (or $c_{11}$ is dispersive but much larger than $c_{66}$ in which case we can set $c_{11}^{-1}=0$ in the following). The approximation to $c_{44}$ in this regime is given by

$$
c_{44}=\frac{B^{2} \epsilon^{2}}{4 \pi \lambda^{2} q_{\perp}^{2}}
$$

This $q$ - dependent elastic modulus changes the nature of the effective long wave-length theory. In Ref. 16, 17, it was argued that such a non-local regime might manifest itself in cases where the translational correlation $\mathrm{ength}_{a}$ was smaller than $\frac{\lambda}{\epsilon}$. The effect of such non-local elasticity on vortex lattices was reexamined recently 34.35 , and the disorder dependence of the Larkin length was computed using a second order FRG calculation. We focus here on the calculation of the translational order parameter. As will be shown below, correlation functions in this regime behave very differently from that of the logarithmic and random manifold regimes.

The disorder independent part of the Hamiltonian when rewritten in terms of the transverse and longitudinal components in Fourier space is

$$
H_{0}=\int \frac{d^{2} q_{\perp} d q_{z}}{(2 \pi)^{3}}\left(c_{66} q_{\perp}^{2}+c_{44} q_{z}^{2}\right) u_{T}^{2}+\left(c_{11} q_{\perp}^{2}+c_{44} q_{z}^{2}\right) u_{L}^{2}
$$

Using the formalism presented in Sec. II and the following connected Green's functions

$$
\begin{aligned}
& G_{c T}^{-1}=c_{44} q_{z}^{2}+c_{66} q_{\perp}^{2} \\
& G_{c L}^{-1}=c_{44} q_{z}^{2}+c_{11} q_{\perp}^{2}
\end{aligned}
$$

we find that $[\sigma]$ in the present case is determined by the following transcendental equation (refer to the Appendix for details)

$$
[\sigma] \log ^{2} \frac{\Lambda^{2}}{[\sigma]}\left[c_{11}^{-3 / 2}+c_{66}^{-3 / 2}\right]=\frac{64 \pi \sqrt{\Gamma}}{K_{0}^{2} T} v
$$

where $\Gamma=B^{2} \epsilon^{2} /\left(4 \pi \lambda^{2}\right)$ and $\Lambda=\frac{2 \pi}{a}$ is the lattice cut-off. Solving (40), we find that $[\sigma]$ has the same form as that given in (15), with the constant $A$ now given by

$$
A_{v}=\frac{64 \pi}{K_{0}^{2} T} \sqrt{\Gamma}\left[\frac{1}{c_{11}^{\frac{3}{2}}}+\frac{1}{c_{66}^{\frac{3}{2}}}\right]^{-1}
$$

Using the result for $[\sigma]$ the displacement correlation functions are given by

$$
\begin{aligned}
\tilde{B}_{L, T}(r, 0)=2 T \int \frac{d^{2} q_{\perp} d q_{z}}{(2 \pi)^{3}} & \cos ^{2}(\theta)\left(1-\cos \left(q_{\perp} r \cos (\theta)\right)\right) \tilde{G}_{L, T} \\
& \left.+\left(1-\cos ^{2}(\theta)\right)\left(1-\cos \left(q_{\perp} r \cos (\theta)\right)\right) \tilde{G}_{T, L}\right]
\end{aligned}
$$

Here

$$
\tilde{G}_{L, T}\left(q_{\perp}, q_{z}\right)=\int_{0}^{1} \frac{d v}{v^{2}} \frac{[\sigma](v)}{G_{c L, T}^{-1}+[\sigma]} G_{c L, T}
$$

Performing a whole lot of cumbersome integrations (see Appendix), we finally obtain the following leading terms in the displacement correlation functions:

$$
\tilde{B}_{L, R}(r, 0)=\tilde{B}(r, 0)=\frac{2}{K_{0}^{2}} \log \log \Lambda^{2} r^{2}
$$

Note that this behaviour of the correlations is exactly analogous to the ones seen earlier in $d=4(\mathrm{cf}$. (19),(31)) and $d=2$ proving again that non-local elastic constants of the type considered here increase the effective dimensionality of the system. Note that despite the strong anisotropy in the hamiltonian, isotropy is restored in the the long wavelength correlation functions in this dispersive regime too. Another interesting point is that these correlations are universal in that they do not depend on the value of any parameter in the theory. The above result can now be used to calculate the translational order parameter. Within the Gaussian approximation it is thus given by: 


$$
\begin{aligned}
O_{T} & =\left\langle\exp i K_{0} \cdot(\mathbf{u}(r)-\mathbf{u}(0))\right\rangle=\exp -\frac{K_{0}^{2}}{2}\left\langle(\mathbf{u}(r, 0)-\mathbf{u}(0,0))^{2}\right\rangle \\
& \simeq \frac{1}{(\ln r)}
\end{aligned}
$$

Note that the decay of $O_{T}$ in the present case is much slower than the $\frac{1}{r}$ decay seen in the case where the elastic constants are local. This implies that the system will look more ordered than it does in the other regimes.

\section{Physical Discussion}

We now estimate the regime of validity of the previous calculation i.e., the range in which the dispersive approximation to $c_{44}$ and hence the $\log \log$ growth of displacements are valid (for a very complete description of the possible regimes in anisotropic superconductors see Ref. 35). Re-analysing the expressions (36) for the elastic constants we see that the approximation (37) to $c_{44}$ is valid provided one is working at length scales $L_{\perp}$ and $L_{z}$ which satisfy the following bounds

$$
\begin{aligned}
& L_{\perp} \ll \lambda / \epsilon \\
& L_{\perp} \ll L_{z} / \epsilon
\end{aligned}
$$

It is also interesting to check when the above dispersive regime includes both the Larkin lengths $R_{\perp}$ and $R_{z}$. The Larkin lengths are determined by the balance between the elastic energy and the disorder which in $d=3$ takes the form

$$
c_{66} r_{f}^{2} R_{z}^{-2} \sim c_{44} r_{f}^{2} R_{\perp}^{-2} \sim r_{f} R_{\perp} R_{z}^{1 / 2} W^{1 / 2}
$$

Here $r_{f}$ is the length over which disorder is correlated. We immediately see that $R_{z} / R_{\perp}=\sqrt{c_{44} / c_{66}}$ and using (37) we thus obtain

$$
R_{z}=\epsilon R_{\perp}^{2} / a
$$

This in conjunction with (48) yields the following bounds on $R_{\perp}$

$$
a \ll R_{\perp} \ll \lambda / \epsilon
$$

The upper limit corresponds to $R_{z}=\lambda^{2} /(\epsilon a)$ and the lower limit $R_{z}=\epsilon a$. The latter may be smaller than the distance $d$ between layers resulting in complicated dimensional crossovers. To stay away from possible dimensional crossover we will restrict $R_{\perp}$ such that

$$
\sqrt{a d / \epsilon} \ll R_{\perp} \ll \lambda / \epsilon
$$

which corresponds to $d<R_{z}<\lambda^{2} /(\epsilon a)$. Note that the above regime becomes non-existent above the "decoupling crossover field" $B_{c r}=\Phi_{0} \epsilon^{2} / d^{2}$.

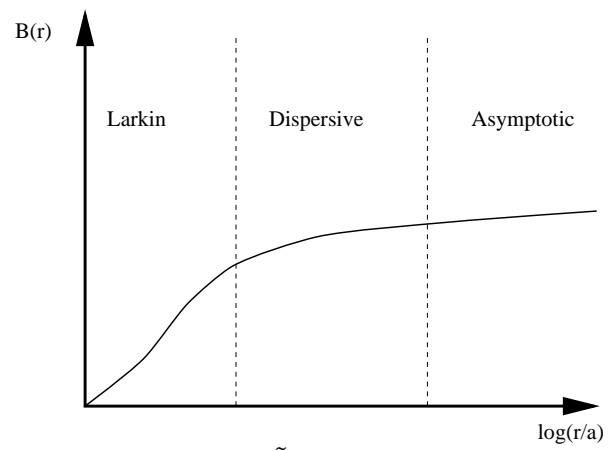

FIG. 2. The growth of the displacements $\tilde{B}(r)$ as a function of $\log (r / a)$ in various regimes. 
We therefore see that the result of (45) can be used at all lengths scales within the bounds prescribed by (48). For asymptotically large scales, the elastic constants become non-dispersive and one expects a crossover to the simple logarithmic growth of displacements discussed in Ref. 16,17. These results are summarised in Fig.2 .

\section{CONCLUSIONS}

We have studied the long wavelength correlations of elastic systems at their upper critical dimension in the presence of weak pointlike disorder. The cases analysed include the simple elastic system at $d=4$, single component systems with $\frac{1}{r}$ interactions and vortex lattices with non-local elastic constants. The systems were studied using the GVM and the equivalence between the GVM and the FRG methods was explicitly demonstrated in the simpler cases of $d=4$ and $d=2$ and we expect such a correspondence to hold in the other cases as well. In all the cases studied we observe the striking fact that the growth of asymptotic displacements is generic in that it is always $\log \log (x)$. This was found to be true even in the case of the anisotropic vortex lattice, where isotropy was recovered in the asymptotic correlation functions. In addition, the prefactor of this log log term (which determines the decay of the translational order within the GVM) is independent of the parameters of the models considered. These systems are again Bragg glasses and the peaks in the diffraction will be more pronounced because the systems are more ordered than their other disordered counterparts which typically show a logarithmic growth of displacements. This feature should be verifiable using decoration and diffraction experiments.

\section{APPENDIX}

We first write down the explicit form of the equation which determines $[\sigma]$ in the case of the vortex lattice described in Sec.III. This is a generalisation of (14) to the two component case and it reads

$$
\sigma(v) \frac{K_{0}^{2} T}{2} \int \frac{d^{d} q}{(2 \pi)^{d}}\left[\frac{1}{\left(G_{c T}(q)^{-1}+[\sigma](v)\right)^{2}}+\frac{1}{\left(G_{c L}(q)^{-1}+[\sigma](v)\right)^{2}}\right]=1
$$

Using the expressions for $G_{c T, L}$ given in (39) and integrating over $q_{\perp}$ and $q_{z}$ we arrive at (40).

Next we present the details of the calculation of the displacement correlation function discussed in Sec.V . Performing the angular integrations in (43) we get

$$
\begin{aligned}
& \tilde{B}_{L}=\frac{2 T}{(2 \pi)^{2}} \int q_{\perp} d q_{\perp} d q_{z} {\left[\left(\frac{1}{2}-J_{0}\left(q_{\perp} r\right)+\frac{J_{1}\left(q_{\perp} r\right)}{q_{\perp} r}\right) \tilde{G}_{L}\right.} \\
&\left.+\left(\frac{1}{2}-\frac{J_{1}\left(q_{\perp} r\right)}{q_{\perp} r}\right)\right] \tilde{G}_{T}
\end{aligned}
$$

$J_{0}$ and $J_{1}$ are the usual Bessel functions and the expressions for $\tilde{G}_{L, T}$ are given in (44). (56) is rewritten as

$$
\tilde{B}_{L}=\frac{2 T}{(2 \pi)^{2}} \int \frac{d v}{v^{2}}\left(I_{1}+I_{2}\right)
$$

where

$$
I_{1}=\int q_{\perp} d q_{\perp} d q_{z}\left[\frac{q_{\perp}^{2}}{\Gamma q_{z}^{2}+c_{11} q_{\perp}^{4}}\right]\left[\frac{q_{\perp}^{2}}{\Gamma q_{z}^{2}+c_{11} q_{\perp}^{4}+[\sigma] q_{\perp}^{2}}\right]\left(\frac{1}{2}-J_{0}+\frac{J_{1}}{q_{\perp} r}\right)
$$

and

$$
I_{2}=\int q_{\perp} d q_{\perp} d q_{z}\left[\frac{q_{\perp}^{2}}{\Gamma q_{z}^{2} 2+c_{66} q_{\perp}^{4}}\right]\left[\frac{q_{\perp}^{2}}{\Gamma q_{z}^{2}+c_{66} q_{\perp}^{4}+[\sigma] q_{\perp}^{2}}\right]\left(\frac{1}{2}-\frac{J_{1}}{q_{\perp} r}\right)
$$

Integrating over $q_{z}$ we get

$$
I_{1}=\frac{\pi}{2 \sqrt{\Gamma c_{11}}} \int q_{\perp} d q_{\perp}\left[\frac{\sqrt{c_{11} q_{\perp}^{2}+[\sigma]}-\sqrt{c_{11}} q_{\perp}}{\sqrt{c_{11} q_{\perp}^{2}+[\sigma]}}\right]\left(\frac{1}{2}-J_{0}+\frac{J_{1}}{q_{\perp} r}\right)
$$


$I_{2}$ has the same form with $c_{11} \leftrightarrow c_{66}$ and the Bessel functions appropriately changed. Integrating with respect to $q_{\perp}$ we now get

$$
\begin{aligned}
I_{1}= & \frac{\pi}{2 \sqrt{\Gamma c_{11}}}\left[\frac{1}{r^{2}}+\frac{[\sigma]}{4 c_{11}} \log 2 \Lambda \sqrt{\frac{c_{11}}{[\sigma]}}\right. \\
& -\frac{1}{2 r} \sqrt{\frac{[\sigma]}{c_{11}}}\left(I_{0}(\alpha) K_{1}(\alpha)-I_{1}(\alpha) K_{0}(\alpha)\right) \\
& \left.-\frac{[\sigma]}{4 c_{11}}\left(I_{0}(\alpha) K_{0}(\alpha)-I_{1}(\alpha) K_{1}(\alpha)\right)\right]
\end{aligned}
$$

and

$$
\begin{aligned}
I_{2}= & \frac{\pi}{2 \sqrt{\Gamma c_{66}}}\left[-\frac{1}{r^{2}}+\frac{[\sigma]}{4 c_{66}} \log 2 \Lambda \sqrt{\frac{c_{66}}{[\sigma]}}\right. \\
& +\frac{1}{2 r} \sqrt{\frac{[\sigma]}{c_{66}}}\left(I_{0}(\beta) K_{1}(\beta)-I_{1}(\beta) K_{0}(\beta)\right)
\end{aligned}
$$

Here $\Lambda$ is the momentum cut-off and the $I$ and $K$ are the modified Bessel functions whose arguments are $\alpha=\frac{r}{2} \sqrt{\frac{[\sigma]}{c_{11}}}$ and $\beta=\frac{r}{2} \sqrt{\frac{[\sigma]}{c_{66}}}$. Finally, adding up the contributions $I_{1}$ and $I_{2}$ we get

$$
\tilde{B}_{L}(r, 0)=\frac{T}{4 \pi \sqrt{\Gamma}} \int \frac{d v}{v^{2}}\left(I_{1}+I_{2}\right)
$$

The transverse correlation function $\tilde{B}_{T}$ is obtained from the above expression by interchanging $c_{11}$ and $c_{66}$. Using the solution for $[\sigma]$ given by $(40)$ and

$$
\tilde{B}_{L}=\frac{A_{v} T}{32 \pi \sqrt{\Gamma}} \int \frac{d v}{v^{2}}\left[\log \frac{A_{v} v}{\Lambda^{2}}\right]^{-2}\left[\frac{E}{2} \log \frac{r^{2} \Lambda^{4}}{A_{v} v}+\mathbf{C} E+\ldots\right.
$$

where $E=c_{11}^{-3 / 2}+c_{66}^{-3 / 2}$. Performing the $v$ integration gives us the result

$$
\tilde{B}_{L, T}(r, 0) \simeq \frac{2}{K_{0}^{2}} \log \log \Lambda^{2} r^{2}
$$

${ }^{1}$ G. Grüner, Rev. Mod. Phys. 60, 1129 (1988).

${ }^{2}$ E. Y. Andrei and al., Phys. Rev. Lett. 60, 2765 (1988).

${ }^{3}$ R. Seshadri and R. M. Westervelt, Phys. Rev. B 46, 5142 (1992).

${ }^{4}$ R. Seshadri and R. M. Westervelt, Phys. Rev. B 46, 5150 (1992).

${ }^{5}$ G. Blatter et al., Rev. Mod. Phys. 66, 1125 (1994).

${ }^{6}$ A. I. Larkin, Sov. Phys. JETP 31, 784 (1970).

${ }^{7}$ A. I. Larkin and Y. N. Ovchinnikov, J. Low Temp. Phys 34, 409 (1979).

${ }^{8}$ T. Nattermann, Phys. Rev. Lett. 64, 2454 (1990).

${ }^{9}$ J. Villain and J. F. Fernandez, Z. Phys. B 54, 139 (1984).

${ }^{10}$ D. S. Fisher, M. P. A. Fisher, and D. A. Huse, Phys. Rev. B 43, 130 (1990).

${ }^{11}$ M. Feigelman, V. B. Geshkenbein, A. I. Larkin, and V. Vinokur, Phys. Rev. Lett. 63, 2303 (1989).

12 M. Charalambous, J. Chaussy, and P. Lejay, Phys. Rev. B 45, 5091 (1992).

${ }^{13}$ H. Safar et al., Phys. Rev. Lett. 70, 3800 (1993).

${ }^{14}$ D. G. Grier et al., Phys. Rev. Lett. 66, 2270 (1991).

${ }^{15}$ E. Zeldov and Al., Nature 375, 373 (1995).

16 T. Giamarchi and P. Le Doussal, Phys. Rev. Lett. 72, 1530 (1994). 
17 T. Giamarchi and P. Le Doussal, Phys. Rev. B 52, 1242 (1995).

18 D. S. Fisher, Phys. Rev. Lett. 56, 1964 (1986).

19 M. Mezard and G. Parisi, J. de Phys. I 1, 809 (1991).

${ }^{20}$ J. P. Bouchaud, M. Mézard, and J. S. Yedidia, Phys. Rev. B 46, 14686 (1992).

${ }^{21}$ S. E. Korshunov, Phys. Rev. B 48, 3969 (1993).

${ }^{22}$ T. Giamarchi and P. Le Doussal, Phys. Rev. B 55, 6577 (1997).

${ }^{23}$ M. J. P. Gingras and D. A. Huse, Phys. Rev. B 53, 15193 (1996).

${ }^{24}$ S. Ryu, A. Kapitulnik, and S. Doniach, Phys. Rev. Lett. 77, 2300 (1996), for an early discussion of the possible phase diagram see also A. Kapitulnik et al. SPIE proceedings 215712 (1994).

25 A. V. Otterlo, R. Scalettar, and G. Zimanyi, cond-mat/9803021.

${ }^{26}$ J. Kierfeld, T. Nattermann, and T. Hwa, Phys. Rev. B 55, 626 (1997).

${ }^{27}$ D. Carpentier, P. Le Doussal, and T. Giamarchi, Europhys. Lett. 35, 379 (1996).

${ }^{28}$ D. S. Fisher, Phys. Rev. Lett. 78, 1964 (1997).

${ }^{29}$ D. Ertas and D. R. Nelson, Physica C 272, 79 (1996).

${ }^{30}$ Y. Y. Goldschmidt, Phys. Rev. B 56, 2800 (1997).

${ }^{31}$ J. Kierfeld, Physica C 300, 171 (1998).

${ }^{32}$ A. E. Koshelev and V. M. Vinokur, Phys. Rev. B 57, 8026 (1998).

${ }^{33}$ V. M. Vinokur and al., Physica C 295, 209 (1998).

${ }^{34}$ H. Bucheli, O. S. Wagner, V. B. Geshkenbein, A. I. Larkin, G. Blatter cond-mat/9801036, to be published in Phys. Rev. B.

${ }^{35}$ O. S. Wagner, Ph.D. thesis, ETH Zürich, 1998.

${ }^{36}$ P. Le Doussal and T. Giamarchi, Phys. Rev. Lett. 74, 606 (1995).

${ }^{37}$ T. Giamarchi and P. Le Doussal, in Statics and dynamics of disordered elastic systems, edited by A. P. Young (World Scientific, Singapore, 1998), p. 321, cond-mat/9705096.

${ }^{38}$ D. Carpentier and P. Le Doussal, Phys. Rev. B 55, 12128 (1997).

${ }^{39}$ D. Carpentier and P. L. Doussal, cond-mat/9712227 to appear in PRL.

${ }^{40}$ R. Chitra, T. Giamarchi, and P. Le Doussal, Phys. Rev. Lett. 80, 3827 (1998).

${ }^{41}$ T. Giamarchi and P. Le Doussal, Phys. Rev. B 53, 15206 (1996).

42 D. R. Nelson and V. M. Vinokur, Phys. Rev. B 48, 13060 (1993). 